\title{
Straßennutzungsgebühren: Eine Lösung zur Vermeidung von Staus?
}

https://doi.org/10.1515/pwp-2019-0039

Zusammenfassung: Angesichts der Zunahme von Staus auf Autobahnen werden die Rufe nach höheren Investitionen in die Fernstraßeninfrastruktur immer lauter. Abhilfe soll vor allem der Ausbau überlasteter Autobahnabschnitte auf sechs oder gar acht Spuren schaffen. Doch der Autobahnausbau verursacht hohe Kosten und erweist sich letztlich häufig als fruchtloses Mittel. In diesem Überblicksbeitrag erörtert Manuel Frondel auf der Grundlage entsprechender Studien aus verschiedenen Ländern als Alternativoption die Einführung von flächendeckenden belastungsabhängigen Gebührensystemen im Autobahnnetz sowie eine Maut zur Vermeidung von immer häufiger auftretenden Verkehrsinfarkten in Städten.

JEL-Klassifikation: R41, D12

Schlüsselwörter: Maut, dynamische Preissetzung

\section{Alltag in der Blechlawine}

Volle Autobahnen, kilometerlange Staus und vergeudete Zeit - das ist immer häufiger tagtägliche Realität für die Autofahrer in Deutschland. Nach Angaben des Allgemeinen Deutschen Automobil-Club (ADAC) gab es im Jahr 2018 auf deutschen Autobahnen so viele Blechlawinen wie nie zuvor: Mehr als 2.000 Staus am Tag hat der ADAC (2019) im Durchschnitt gezählt. In der Summe kam der Verkehrsclub auf rund 745.000 - und damit auf ein Plus von rund 3 Prozent im Vergleich zu 2017. Die gemeldeten Staulängen wuchsen um rund 5 Prozent und summierten sich auf etwa 1,5 Millionen Kilometer. Das ist ein dramatischer Anstieg innerhalb weniger Jahre: Im Jahr 2011 wurden rund 189.000 Staus auf deutschen Autobahnen gemeldet, und die Staus summierten sich zu einer Gesamtlänge von etwa 0,45 Millionen Kilometer (Intraplan 2011, S. 1). Nordrhein-Westfalen ist dabei das Bundesland mit den

\footnotetext{
*Kontaktperson: Manuel Frondel, RWI Leibniz-Institut für Wirtschaftsforschung und Ruhr-Universität Bochum, Hohenzollernstr. 1-3, 45128 Essen, E-Mail: frondel@rwi-essen.de
}

häufigsten und längsten Staus, nicht viel weniger betroffen sind Baden-Württemberg, Bayern, Hessen und Niedersachsen.

Für die Zunahme an Staus nannte der ADAC (2019) zwei Gründe: Zum einen gab es 2018 auf den Autobahnen rund 3 Prozent mehr Baustellen. Zum anderen ist die Fahrleistung, die jedes Auto im Jahr zurücklegt, um 0,4 Prozent gestiegen. Darüber hinaus nimmt die Zahl der Autos in Deutschland beinahe fortwährend $\mathrm{zu}$ : allein zwischen 2007 und 2018 nach Angaben des Kraftfahrtbundesamtes (KBA) um mehr als 14 Prozent, von 41,2 auf 47,1 Millionen (KBA 2019). Wenn zudem alle Autos im Durchschnitt noch einige Kilometer mehr gefahren werden, wird es auf den Straßen automatisch enger, sofern die Kapazitäten nicht entsprechend ausgebaut werden.

Aussicht auf Besserung besteht nach Ansicht des ADAC nicht. Im Gegenteil: Intraplan Consult prognostiziert in einer 2011 veröffentlichten Studie im Auftrag des ADAC, dass bis zum Jahr 2025 die überlasteten Autobahnabschnitte angesichts immer größerer Verkehrsmengen auf insgesamt 2.000 Kilometer anwachsen könnten (Intraplan 2011). Im Jahr 2010, so heißt es in der Studie, betrug die betroffene Netzlänge rund 1.600 Kilometer. Für die Prognose ging Intraplan nicht nur von der Fertigstellung zahlreicher Neu- und Ausbauprojekte bis zum Jahr 2025 aus, sondern auch von einer 10-prozentigen Kapazitätserhöhung für alle Autobahnabschnitte.

Die im Stau verbrachten Stunden kosten nicht nur Zeit. Der Verkehrsdatenanbieter INRIX (2018) errechnete für Deutschland für das Jahr 2017 Staukosten von rund 80 Milliarden Euro, wobei auch Staus in Städten einbezogen wurden. ${ }^{1}$ Die Kosten von Staus beinhalten vor allem die

1 INRIX (2018) analysiert in der INRIX 2017 Traffic Scorecard die Auswirkungen von Verkehrsstaus in 1.360 Städten und 38 Ländern aus aller Welt. Es handelt sich um die umfangreichste Studie dieser Art. Von zentraler Bedeutung für die Schätzung der Kosten von Staus sind die zugrunde gelegten Annahmen (Eisenkopf 2018). Wegen des dominanten Anteils von Personenwagen mit privater Nutzung an den Fahrleistungen ist hierfür der durchschnittliche Kostensatz wesentlich, mit dem jede persönliche Stunde im Stau bewertet wird. In früheren Studien ist man von durchschnittlichen ökonomischen Verlusten in der Höhe von 15 bis 20 Euro je Staustunde ausgegangen. 
Opportunitätskosten in Form der im Stau verbrachten Zeit. Hinzu kommen im Wesentlichen noch die Kosten des Kraftstoffmehrverbrauchs und die gesellschaftlichen Kosten höherer Emissionen.

Vor diesem Hintergrund forderte der ADAC (2019) einmal mehr, die Investitionen in die Fernstraßeninfrastruktur zu erhöhen. Abhilfe soll vor allem der sechs- bzw. achtstreifige Ausbau überlasteter Autobahnabschnitte schaffen. Doch auch der Autobahnausbau verursacht hohe Kosten: Abhängig beispielsweise vom Gelände sind dafür je Kilometer grob 10 bis 20 Millionen Euro zu veranschlagen; in Einzelfällen, vor allem wenn Tunnel oder Talbrücken gebaut werden müssen, können die Kosten je Kilometer sogar deutlich über 100 Millionen Euro liegen (Statista 2019). Hinzu kommen Folgekosten für Natur und Umwelt durch die Umweltzerstörung sowie die Beeinträchtigung des Landschaftsbildes.

Viel schwerer wiegt jedoch, dass sich der Ausbau der Straßeninfrastruktur, obwohl auf den ersten Blick die naheliegende Lösung für Stauprobleme, letztendlich als fruchtloses Mittel erweist (Hsu und Zhang 2014, S. 65). Denn die Erhöhung der Verkehrsgeschwindigkeit, die sich dank der Kapazitätserweiterung ergibt, zieht neuen Verkehr an bzw. bringt den zuvor verhinderten Verkehr zurück auf die Straße. Im Ergebnis sind die ausgebauten Straßen wieder ebenso verstopft wie zuvor. Dieses paradoxe Ergebnis bezeichnet man in Anlehnung an Anthony Downs $(1962,1992)$ als fundamentales Verkehrsstaugesetz. Theoretisch folgt daraus, dass die Elastizität des Straßenverkehrs in Bezug auf Kapazitätserweiterungen mindestens 1 betragen sollte.

Duranton und Turner (2011) ziehen aus diesen Befunden und ihren eigenen empirischen Ergebnissen für die Vereinigten Staaten, nach denen die Summe der Fahrzeugkilometer mit der Länge der Highways wächst, die Schlussfolgerung, es sei wenig wahrscheinlich, dass der Ausbau von Straßen helfe, Verkehrstaus zu beseitigen: „Our results strongly support the hypothesis that roads cause traffic“ (Duranton und Turner 2011, S. 2618). Tatsächlich haben in Städten wie Los Angeles und Houston sogar Milliardeninvestitionen in neue Straßen wenig dazu beigetragen, die Pendelzeiten zu verringern (Cramton, Geddes und Ockenfels 2019, S. 127).

Im Folgenden erörtere ich als Alternativinstrument zum Straßenausbau die Einführung von flächendeckenden belastungsabhängigen Gebührensystemen im Autobahnnetz sowie eine Städte-Maut zur Vermeidung der immer

INRIX beziffert hingegen die gesamten Kosten je Staustunde auf 59 Euro. häufiger auftretenden Verkehrsinfarkte auf Autobahnen und in Städten. Dies lehnt sich an Vickrey $(1963,1969)$ an, der schon vor langer Zeit eine dynamische, also belastungsabhängige Bepreisung knapper Straßenkapazitäten zur Vermeidung von Verkehrstaus empfahl. Zunächst werden Stauursachen und dynamische Straßennutzungsgebühren als mögliche Lösung für Stauprobleme beschrieben (Abschnitt 2), dann die Erfahrungen mit Straßennutzungsgebühren in den Vereinigten Staaten (Abschnitt 3) und anschließend die empirische Evidenz zu den Effekten von Städte-Mauts, die in einigen europäischen Städten bereits seit Jahrzehnten existieren (Abschnitt 4). Es folgt ein Fazit zu den Wirkungen nutzungsabhängiger Gebührensysteme im Hinblick auf die Vermeidung von Staus (Abschnitt5).

\section{Ursachen von Verkehrsstaus und dynamische Preissetzung als Lösungsansatz}

Staus und exzessive Nutzung von Personenwagen haben ihre Ursachen nicht zuletzt darin, dass Autofahrer weder die externen Kosten für die von ihnen verursachten Umweltschäden zu tragen haben, noch die Kosten, die sie anderen Autofahrern durch ihre Straßennutzung aufbürden. Dadurch gerät das Autofahren zu günstig. Im Kern sind Verkehrsstaus somit das Ergebnis einer mangelnden Internalisierung externer Kosten und auf das Fehlen eines Mechanismus zurückzuführen, der hilft, die vorhandenen Straßenkapazitäten effizient zu nutzen (Federal Highway Administration, FHWA 2006, S. 1).

Dieses Koordinierungsproblem lässt sich nach Auffassung von Ökonomen durch eine dynamische Bepreisung knapper Straßenkapazitäten lösen (vgl. z. B. Cramton, Geddes und Ockenfels 2018). Wenn für die Straßen, die zu manchen Zeiten stark befahren sind, belastungsabhängige Nutzungspreise eingeführt werden, setzt dies Anreize für die Autofahrer, ihr Verhalten kurzfristig wie folgt anzupassen: (1) Sie verschieben die Fahrzeiten auf andere, weniger staukritische Tageszeiten; (2) sie nutzen andere Verkehrsmittel oder Verkehrswege; (3) sie steigen um auf eine gemeinschaftliche Nutzung von Fahrzeugen; oder (4) sie verzichten auch einmal ganz auf Fahrten.

Eine solche dynamische Bepreisung würde durchaus nicht alle Fahrer abschrecken und die Straßen wären infolgedessen auch keinesfalls leergefegt. Im Gegenteil: Eine dynamische Bepreisung nutzt aus, dass ein Teil der Straßennutzer zu Stoßzeiten keine Pendler sind und dass infolge der Bepreisung bereits die Verringerung eines kleinen Teils an Nutzern zu einer effizienteren Nutzung der 
Straßenkapazität, also einem höheren Durchsatz an Fahrzeugen führt (FHWA 2006, S. 1). Wie bei Erreichen der Kapazitätsgrenzen bereits ein kleiner Anstieg der Zahl der Fahrzeuge zu einer drastischen Reduktion der Durchschnittsgeschwindigkeit und zum Stau führt (Cramton, Geddes und Ockenfels 2019, S. 176), führt umgekehrt die Verringerung eines Bruchteils an Nutzern zu Stoßzeiten zu einem deutlichen Anstieg der Durchschnittsgeschwindigkeit und verringert die Wahrscheinlichkeit des Auftretens eines Staus massiv. Tatsächlich gibt es empirische Evidenz dafür, dass eine dynamische Bepreisung den Durchsatz von Personenwagen zu Stoßzeiten auf ansonsten verstopften Straßen verdoppelt (FHWA 2006, S. 3).

Mit Hilfe derzeit verfügbarer Technologien, mit denen der Aufenthaltsort eines Fahrzeugs bis auf wenige Zentimeter nachverfolgt werden kann, ist eine dynamische Bepreisung in Echtzeit heute bereits möglich. So könnten Straßennutzungsgebühren je nach Verkehrsaufkommen und Straße häufig über die Zeit hinweg geändert werden, ohne dass den Fahrern ein zusätzlicher Zeitaufwand entsteht, wie ihn beispielsweise ein Stopp am Mauthäuschen auf der Autobahn nach sich zieht. Hierzu können zum Beispiel Transponder eingesetzt werden, die wenige Euro kosten und mittels Antennen abgelesen werden. In den Vereinigten Staaten werden die Straßennutzungsgebühren auf diese Weise bislang am häufigsten erhoben (FHWA 2006, S. 2).

Obgleich mit mehreren hundert Euro deutlich teurer, könnten im Fahrzeug fest installierte Geräte, die auf Basis des Global Positioning System (GPS) funktionieren, künftig die am häufigsten eingesetzte Technologie darstellen. Den höheren Kosten stehen zusätzliche Dienstleistungen gegenüber, zum Beispiel ein Navigationssystem, und im Vergleich zu den Anschaffungskosten eines Neuwagens dürften sie nur wenig ins Gewicht fallen. Dafür würde es diese Technologie erlauben, maßgeschneiderte Gebühren für jedes einzelne Fahrzeug je nach Schadstoffausstoß und Verkehrssituation $\mathrm{zu}$ erheben. Für schadstoffintensive Fahrzeuge, insbesondere Lastwagen, wäre mehr zu bezahlen, die Gebühren für Elektrofahrzeuge ließen sich niedriger ansetzen. So entstünde mehr Platz für schadstoffärmere Fahrzeuge auf den Straßen. Die Kehrseite der ökologischen Effizienz indes wäre verteilungspolitischer Natur: Die Mitglieder einkommensschwächerer Haushalte, die tendenziell ältere und schadstoffintensivere Fahrerzeuge fahren, könnten sich benachteiligt fühlen.

Auf GPS basierende Geräte werden schon heute in zahlreichen Ländern zur Mauterhebung von Lastwagen eingesetzt, beispielsweise in Deutschland, Österreich und der Schweiz. Da die Mauttarife jedoch fix sind und nicht nutzungsabhängig variieren, helfen sie nicht bei der Ver- meidung von Staus. Dazu müssten die Mauttarife in Abhängigkeit von Angebot und Nachfrage sinnvoll schwanken. Solche variablen Nutzungspreise werden bereits erfolgreich in vielen anderen Bereichen eingesetzt, zum Beispiel im Verkauf von Flugtickets, im Zusammenhang mit Telefontarifen und auf den restrukturierten Strommärkten der Vereinigten Staaten, wo man die Kapazitäten des Stromnetzes dadurch effizient bepreist, dass Strom zu jeder Zeit und an jedem Ort einen individuellen Preis erhält. Diese Preise werden als lokale Grenzpreise bezeichnet. Unter Ökonomen herrscht große Einigkeit, dass eine dynamische Nutzungsbepreisung auch im Straßenverkehr der praktikabelste und zugleich nachhaltigste Ansatz ist, um Verkehrsstaus dauerhaft zu vermeiden (FHWA 2006, S. 1). Damit würden knappe Straßenkapazitäten zu einem gewöhnlichen Gut, das man verkaufen und kaufen kann; der Verkehr würde, zum größtmöglichen Nutzen der Straßennutzer, zu einem Markt. Insgesamt ließe sich so der Nutzen der Straßeninfrastruktur maximieren (Cramton, Geddes und Ockenfels 2019).

\section{Erfahrungen mit Straßennutz- ungsgebühren aus den Vereinigten Staaten}

Um innovative Ansätze zur Verringerung von Staus zu fördern, rief das amerikanische Verkehrsministerium im vergangenen Jahrzehnt das Programm „Urban Partnership Agreement (UPA) and Congestion Reduction Demonstration (CRD)“ ins Leben. Dieses lässt Metropolregionen, die sich auf einen koordinierten Ansatz zur Bekämpfung von Staus festlegen, finanzielle Förderung und technische Hilfe zukommen. Dabei wurde viel Wert auf die Evaluation der Wirkungen gelegt. So förderte das Ministerium als wesentlichen Baustein der Evaluierung eine Vorher-Nachher-Erhebung des Mobilitätsverhaltens von Haushalten in Seattle und Atlanta. Dies sind zwei von sechs Metropolregionen, die mit diesem Programm unterstützt wurden.

Das Kernstück des UPA-Projekts in Seattle war die Erhebung einer variablen, tageszeitabhängigen Gebühr für die Nutzung der Evergreen-Point-Brücke, welche die State Route SR-520 nahe der Innenstadt über den WashingtonSee führt. Parallel dazu bietet die Interstate I-90 eine gebührenfreie Alternativroute zur SR-520, die ebenfalls den Washington-See quert. Vor der Einführung der Nutzungsgebühren im Dezember 2011 gab es auf der SR-520 in beiden Richtungen massive Staus während vieler Stunden des Tages. Investitionen in den öffentlichen Nahverkehr und in Maßnahmen zum Verkehrsmanagement begleite- 
ten die Einführung der Gebühren; die Einnahmen fließen in die Instandhaltung der Brücke .

Peirce et al. (2014) haben die Effekte der Nutzungsgebühr in einer empirischen Studie mit einem zweistufigen Paneldatenansatz analysiert. Die dafür nötigen Daten wurden in zwei Erhebungen gewonnen: Die erste Erhebung fand vor Einführung der Nutzungsgebühren im Herbst 2010 statt, die zweite Erhebung nach deren Einführung im Frühjahr 2012. In beiden Erhebungswellen führten mehr als 3.600 Teilnehmer aus rund 2.000 Haushalten über zwei Tage hinweg ein Wegetagebuch und machten zusätzliche Angaben zu ihren Fahrten im Korridor des WashingtonSees sowie zu ihrem allgemeinen Verkehrsverhalten und ihren persönlichen Einstellungen.

$\mathrm{Zu}$ den Kernergebnissen der Studie zählt, dass es zu einem markanten Rückgang der Anzahl an Fahrten auf der SR-520 von 43 Prozent gekommen ist. Dazu beigetragen hat eine Verlagerung des Verkehrs auf die gebührenfreie Alternativroute I-90, auf die etwa ein Viertel der früheren SR-520-Nutzer wechselten. Am häufigsten taten dies einkommensschwache Haushalte und Personen mit geringer Arbeitszeitflexibilität. Die Effekte der Nutzungsgebühr für die SR-520 variierten stark mit dem Fahrzweck: Die Menschen drosselten ihre Fahrten zur Schule und zum Einkaufen signifikant, ihre Fahrten zur Arbeit und für Freizeitzwecke jedoch weniger stark. In der zweiten Erhebung zeigte sich ein starker Anstieg der Zufriedenheit mit dem Pendeln, der Fahrgeschwindigkeit und der Verlässlichkeit der Nutzung der SR-520. Die persönliche Einstellung zur Nutzungsgebühr verbesserte sich leicht.

Variable Gebühren für die Nutzung von Brücken haben in den Vereinigten Staaten eine lange Tradition (FHWA 2019): Bereits seit August 1998 werden Gebühren für die Nutzung der Midpoint- und Cape-Coral-Brücken in Lee County, Florida erhoben. Um die Nutzung der Brücken von Stoßzeiten in andere Zeiten zu verlagern, wird Brückennutzern ein Rabatt von 50 Prozent angeboten, wenn sie die Brücken in den Zeiten mit geringerer Verkehrsdichte zwischen 6:30 und 7:00 Uhr, 9:00 und 11:00 Uhr, 14:00 und 16:00 Uhr oder zwischen 18:30 und 19:00 Uhr benutzen und die Gebühren elektronisch abbuchen lassen.

Neben den Nutzungsgebühren für Brücken gibt es in den Vereinigten Staaten bislang hauptsächlich einen weiteren Typ von Nutzungspreisstrategien: die mit der Tageszeit variierende Bepreisung von getrennten Fahrspuren auf Highways, Express oder High-occupancy toll lanes genannt. Solche „HOT lanes“ werden derzeit in San Diego, Minneapolis, Denver, Houston und Salt Lake City betrieben, Express toll lanes zum Beispiel in Orange County in Kalifornien. So ist seit 1998 für Fahrzeuge, die mit nur einer Person besetzt sind, bei Benutzung der HOT lanes der I-15 in San Diego eine vom Verkehrsaufkommen abhängige Gebühr zu bezahlen. Infolgedessen stieg die Zahl der mit mehr als einer Person besetzten Autos zwischen 1998 und 2006 um rund 50 Prozent. Die Gebühren steigen oder fallen alle sechs Minuten um jeweils 25 Cent, um den Verkehrsfluss nicht ins Stocken geraten zu lassen. Etwa die Hälfte der seit 1998 durch diese Gebühr entstandenen Einnahmen in Höhe von mehr als 7 Millionen Dollar flossen in die Verbesserung des Verkehrs im Korridor von San Diego. Die HOT lanes genießen eine überwältigende Unterstützung unter den Nutzern der I-15, einschließlich der Nutzer der HOT lanes.

Trotz positiver Erfahrungen mit belastungsabhängigen Straßengebühren in den Vereinigten Staaten gibt es noch einige große Herausforderungen (Cramton, Geddes und Ockenfels 2018, S. 24), die es zu bewältigen gilt, bevor die Einführung von belastungsabhängigen Gebührensystemen andernorts, insbesondere im deutschen Autobahnnetz, Realität werden kann. Erstens bezeichnen Kritiker die Erhebung von Straßennutzungsgebühren oftmals als sozial ungerecht. Einkommensschwache Haushalte seien davon stärker betroffen als wohlhabende Haushalte. Dieser Einwand lässt sich wohl nur durch einen verstärkten Ausbau des öffentlichen Nah- und Fernverkehrs noch vor der Einführung von Straßennutzungsgebühren entkräften. Zweitens bedarf die Technologie zur automatischen Erhebung von Straßennutzungsgebühren weiterer Verbesserungen; vor allem sollte sie kostengünstiger werden. Damit sie eines Tages länderübergreifend einsetzbar wird, sollte sie zudem standardisiert werden. Drittens sollte die Erfassung hohen Datenschutzanforderungen genügen, damit sie Akzeptanz finden kann. Viertens sollten die bei einer dynamischen Bepreisung erhobenen Daten mit immer besseren statistisch-ökonomischen Modellen verarbeitet werden, um den Verkehrsfluss zu optimieren.

Vor dem Hintergrund dieser Herausforderungen muss sich die Begeisterung von belastungsabhängigen Gebühren bislang stark in Grenzen halten, insbesondere wenn man darin eine Kur gegen die immer häufiger auftretenden Verkehrsinfarkte auf deutschen Autobahnen sehen möchte. Ein erster wichtiger Schritt in Richtung einer höheren Akzeptanz nutzungsabhängiger Gebühren könnte jedoch die erfolgreiche Einführung einer Städte-Maut sein, einer Gebühr für eine Fahrt mit dem Auto in eine Stadt, um die vielfältigen Verkehrsprobleme in vielen deutschen Großstädten zu lösen. 


\section{Effekte einer Städte-Maut}

Weil es in Deutschland immer mehr Autos gibt, nehmen die durch den Verkehr verursachten Probleme auch in den Städten weiter zu: Neben Lärm, Feinstaub und Stickoxidemissionen verursachen durch parkende Autos überfüllte Innenstädte und durch Staus verstopfte Straßen massive negative externe Effekte. Um die volkswirtschaftlichen Kosten des Autoverkehrs verursachergerecht zuzuordnen, wäre eine nutzungsabhängige Finanzierung der Verkehrsinfrastruktur auch in Städten sinnvoll. Mit der Einführung einer Städte-Maut könnten der öffentliche Raum und die öffentliche Infrastruktur in Städten effizienter genutzt werden. Insbesondere die verkehrsbedingten lokalen Schadstoffemissionen ließen sich effizient bekämpfen (Achtnicht, Kesternich und Sturm 2018). Der jeweils zu entrichtende Mautbetrag sollte dabei nach dem Schadstoffausstoß des Personenwagens gestaffelt sein. Die negativen Auswirkungen des Autofahrens, unter denen die Stadtbewohner besonders zu leiden haben, würden damit verringert, wie die folgende empirische Evidenz zeigt.

In Städten wie Oslo, Trondheim, Bergen, Stockholm, Göteborg, Mailand, Palermo, Bologna, London und Singapur setzt man teilweise schon seit Jahrzehnten auf eine Städte-Maut. In Stockholm zum Beispiel, wo seit dem Jahr 2006 - zunächst für eine Probezeit von 7 Monaten - eine uhrzeitabhängige Maut für die Einfahrt in die Innenstadt erhoben wird, ging der Autoverkehr in den Jahren 20062011 um 18 bis 21 Prozent gegenüber dem Jahr 2005 zurück (Börjesson et al. 2012). Die Maut wird in Stockholm von Montag bis Freitag in der Zeit von 6:30 bis 18:29 fällig. In den Stoßzeiten von 7:30 bis 8:30 und 16:00 bis 17:30 sind 35 Kronen (ca. 3,30 Euro) zu zahlen, in den übrigen Stunden ist die Maut günstiger, der geringste Mautbetrag liegt bei 15 Kronen (ca. 1,40 Euro). Die Maut wird in beiden Richtungen fällig, das heißt eine Fahrt in die Stadt und zurück kostet zu Stoßzeiten 70 Kronen (ca. 6,60 Euro). Der Höchstbetrag bei mehrmaligem Fahren in die Mautzone liegt bei 105 Kronen bzw. rund 9,90 Euro am Tag. Die Mautgebühren sind im Nachhinein zu bezahlen und werden dem Fahrzeughalter am Ende des Folgemonats in Rechnung gestellt. Zur Registrierung wird das Fahrzeugkennzeichen beim Passieren einer Kontrollstation im Innenstadtbereich fotografiert und so der Fahrzeughalter ermittelt.

Die probeweise Einführung der Innenstadtmaut in Stockholm im Januar 2006 ermöglichte der Wissenschaft nahezu ideale Bedingungen zur Evaluierung der Auswirkungen der Maut. Während der siebenmonatigen Testphase maßen Mitarbeiter der Stadt Stockholm kontinuierlich die Verkehrsstärke, die Luftqualitätswerte, die Fahrzeit, die Einflüsse auf den Parkplatzsuchverkehr sowie den Radverkehr. Es ließ sich dadurch eindeutig feststellen, dass die nachweisbare Verkehrsumlenkung mit der Maut zusammenhing (vgl. Beser Hugosson, Sjöberg und Byström 2006). Ein augenscheinliches Indiz dafür ist, dass nach Ende der Testphase die Verkehrsmenge wieder auf den ursprünglichen Wert stieg.

In Abbildung 1 sind die Veränderungen der Verkehrsmengen gut zu erkennen: Die schwarze Linie stellt das Verkehrsaufkommen vor Einführung der Maut dar, die grüne Linie den Zustand danach. Die Reduzierung des Verkehrsaufkommens ist je nach Uhrzeit unterschiedlich und lag in der Mautzeit im Durchschnitt bei 22 Prozent (Beser Hugosson, Sjöberg und Byström 2006, S. 6). Die morgendliche Spitze wurde um 16 Prozent reduziert, die abendliche Spitze um 24 Prozent. Über die gesamten 24 Stunden eines Tages betrachtet, war das Verkehrsaufkommen im April 2006 um 19 Prozent geringer als vor Einführung der Maut im April 2005. Dies entspricht einem Rückgang von rund 100.000 Fahrten am Tag. Außerhalb der Mautzeiten blieb das Verkehrsaufkommen weitgehend unverändert. Dies zeigt, dass die viele Fahrten nicht in die mautfreien Zeiten verlagert wurden, sondern ganz unterblieben. Dass zahlreiche Autofahrer auf die finanziellen Anreize reagierten, die durch die Maut gesetzt wurden, sieht man besonders gut am Ende der Mautzeit um 18:29 Uhr. Danach steigt das Verkehrsaufkommen sprunghaft an. Offenbar warten Autofahrer bis 18:30 Uhr, um in der mautfreien Zeit die Stadt wieder zu verlassen, und arbeiten bis dahin oder nutzen die Zeit im Zentrum anderweitig, beispielsweise zum Einkaufen.

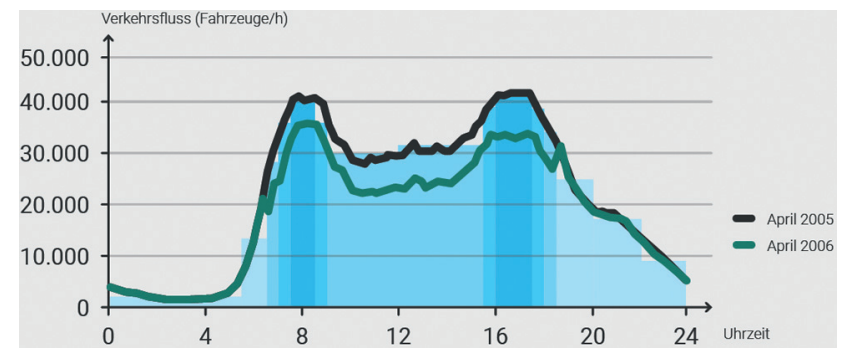

Abbildung 1: Verkehrsfluss in Stockholm im Tagesverlauf im April 2005 und im April 2006

Quelle: Beser Hugosson, Sjöberg und Byström 2006, S. 30

Der unterschiedliche Rückgang des Verkehrsaufkommens in Stockholm zu verschiedenen Tageszeiten zeigt, dass man mit einer auslastungsabhängigen Maut Verkehrsströme in gewissem Maße steuern kann. Diese Steuerung kann jedoch noch verbessert werden: Mit den bereits heute verfügbaren automatischen Bezahlsystemen wäre es möglich, Aufwand und Kosten der Erfassung deutlich zu reduzie- 
ren, sodass die Nettoeinnahmen für die Städte höher ausfielen als mit den bestehenden Erfassungssystemen für die Kennzeichen von Personenwagen. Zudem würden es technisch versierte Systeme sogar erlauben, eine vom jeweiligen Fahrzeugtyp abhängige individuelle Maut zu erheben und auf dieser Basis eine weitaus stärkere Differenzierung als bislang vorzunehmen, zum Beispiel nach Schadstoffklassen.

Ein nach Uhrzeiten gestaffeltes Mautsystem wie in Stockholm gibt es seit dem Jahr 2013 auch in Göteborg. Ebenso wie in Stockholm wird in Göteborg die Maut von Montag bis Freitag in der Zeit von 6:00-18:29 Uhr erhoben; mautfrei sind die Wochenenden, Feiertage, der Tag vor einem Feiertag sowie der Monat Juli. Zu den Hauptverkehrszeiten am Morgen und am Nachmittag wird ein höherer Betrag von 22 Kronen (ca. 2,10 Euro) erhoben. Der jeweils zu zahlende Betrag ist an den Mautstationen abgebildet. Der Höchstbetrag bei mehrmaligem Passieren der Mautzonengrenze liegt bei 60 Kronen (ca. 5,70 Euro) am Tag. Acht Monate nach Einführung der Maut pendelte sich die Reduktion des Personenwagen-Verkehrs während der Mautzeiten bei 12 Prozent ein (Börjesson und Kristoffersson 2015); der Rückgang fiel während der Stoßzeiten am Morgen leicht höher aus. Außerhalb der Mautzeiten blieb das Verkehrsaufkommen hingegen weitgehend unverändert.

Ein weiteres Beispiel ist London. Dort wird seit Februar 2003 von Montag bis Freitag zwischen 7:00 und 18:00 Uhr eine Maut erhoben. Anfänglich betrug sie 5 Pfund am Tag, aktuell sind es 11,5 Pfund (TfL 2019) bzw. rund 13 Euro. Wie in Göteborg und Stockholm werden die Kennzeichen der Autos an den Einfahrtstraßen registriert. Regelmäßige Nutzer bezahlen per Kontoabbuchung oder in Form einer verbilligten Vorauszahlung mit Mengenrabatt. Bei Zahlungsverzug werden empfindliche Strafen in Höhe von 160 Pfund fällig, wobei sich die Strafe auf die Hälfte reduziert, wenn man innerhalb einer bestimmten Frist bezahlt. Die Einnahmen aus dieser Londoner „Congestion charge“ mussten in den 10 Jahren nach ihrer Einführung von Gesetzes wegen in Verbesserungen der Nahverkehrsinfrastruktur investiert werden. Der Verkehrsgesellschaft Transport for London (TfL) blieb nach Abzug der Betriebskosten regelmäßig ein dreistelliger Millionenbetrag. Bis Mitte 2005 sank die Zahl der Fahrten in Personenwagen um rund ein Drittel (Leape 2006). Im Gegenzug nahm die Zahl der Busfahrgäste um rund 38 Prozent zu; etwa die Hälfte dieses Anstieges führt Leape auf die Maut, den Rest auf eine Verbesserung der Busverkehrsdienstleistungen zurück. Allerdings hat der Personenwagen-Verkehr in den vergangenen Jahren wieder sein $\mathrm{Ni}$ veau aus der Zeit vor Einführung der Maut erreicht, und es stellt sich die Frage, ob dies ein spezielles Londoner Phänomen ist oder ob damit auch in anderen Städten zu rechnen ist.

Dem ist wohl nicht so, denn die Erfahrungen aus Stockholm, Göteborg und anderen Städten zeigen weit überwiegend, dass mit einer Städte-Maut das Pkw-Aufkommen deutlich gesenkt werden kann. Damit verringert sich sowohl die Menge der Emissionen als auch die Häufigkeit von Staus. Diese Wirkungen entfaltet eine Städte-Maut dadurch, dass die Kosten für eine Autofahrt in die Stadt im Vergleich zu den Alternativen steigen. Das erhöht die Attraktivität der Nutzung anderer Verkehrsmittel und führt zu einem Umstieg auf Verkehrsmittel, mit denen weniger negative externe Effekte verbunden sind, zum Beispiel den öffentlichen Nahverkehr (ÖPNV) oder das Fahrrad. Eine Städte-Maut regt außerdem zu einer effizienteren Nutzung von Personenwagen an, beispielsweise dadurch, dass Pendler Fahrgemeinschaften bilden.

Eine Städte-Maut würde den Städten und Kommunen zusätzliche Einnahmen bescheren, die zweckgebunden in den Ausbau des öffentlichen Personennahverkehrs und der Rad- und Fußwege sowie in die Finanzierung von Sozialtickets fließen könnten. Es gäbe somit mehr, bessere und günstigere Optionen für die tägliche Mobilität. Wenn sich die Verantwortlichen in der Kommunalpolitik bei der Festsetzung der Städte-Maut an jenen Beträgen orientierten, die andernorts in Europa erhoben werden, dann handelte es sich für jeden Autofahrer um wenige Euro zusätzlich am Tag. Dies liegt in der Größenordnung der Parkgebühren, die man üblicherweise in städtischen Parkhäusern zu entrichten hat. Eine Städte-Maut ist anderen Regulierungsinstrumenten, vor allem Fahrverboten, deutlich überlegen, auch weil den Menschen dadurch ihre Wahlfreiheit bewahrt würde und sie frei entscheiden könnten, ob ihnen die Fahrt ins Zentrum mit dem eigenen Personenwagen so viel wert ist, wie sie an Betriebskosten, vor allem für Sprit, sowie an Park- und Mautgebühren, aufzubringen hätten.

Die in Deutschland diskutierten oder bereits erlassenen Fahrverbote lassen vielen Menschen diese Wahlfreiheit hingegen nicht. Vielmehr haben sie den Nachteil, dass sie bestimmte Fahrzeuge vom Verkehr ausschließen, ohne dass dabei die sozialen Folgen berücksichtigt oder Alternativoptionen angeboten werden. Eine Städte-Maut hingegen beließe insbesondere Haushalten mit niedrigen Einkommen, die beispielsweise nur über einen älteren DieselPersonenwagen verfügen, einen größeren Handlungsspielraum als kategorische Fahrverbote. 


\section{Fazit: Ein Ausweg aus dem Verkehrskollaps}

Eine entfernungs-, zeit- und schadstoffabhängige Straßennutzungsgebühr, die zeitlich fein gestaffelt alle negativen externen Effekte des Verkehrs wie Stau, Lärm und Luftschadstoffe berücksichtigt, wäre das aus ökonomischer Sicht am besten geeignete Instrument, um die volkswirtschaftlichen Kosten der Autonutzung verursachergerecht zuzuordnen. Darüber hinaus wäre eine adäquate Bepreisung öffentlicher Parkplätze in Städten die aus ökonomischer Perspektive beste Möglichkeit, um die Überlastung von Parkflächen zu reduzieren.

Effiziente Straßennutzungsgebühren hätten viele Vorteile. Sie würden den Umwelt- und Ressourcenverbrauch eindämmen, da infolge eines effizienteren Verkehrsflusses die Schadstoff- und Treibhausgasemissionen abnähmen. Darüber hinaus würden sie den Verkehrsfluss verstetigen und besser vorhersagbar machen, was die Verkehrssicherheit erhöhte. Eine dynamische Bepreisung würde zudem wertvolle Informationen liefern, um knappe Investitionsmittel in jene Projekte zu lenken, welche die Straßennutzer am meisten schätzen. Zugleich würde eine dynamische Bepreisung die für die Erhaltung und den weiteren Ausbau der Straßen nötigen finanziellen Mittel liefern (Cramton, Geddes und Ockenfels 2019, S. 127).

Daneben gibt es weitere Vorteile: (1) Die knappen Straßenkapazitäten würden gerade jenen Autofahrern zur Verfügung stehen, die diese zu bestimmten Tageszeiten am meisten zu schätzen wissen und entsprechend hohe Gebühren bezahlen. (2) Diese Nutzungsgebühren könnten die sozialen Kosten des Straßenverkehrs besser reflektieren als Kraftstoffsteuern. (3) Dadurch würden Anreize für technologische Innovationen gesetzt, mit denen die Nachfrage nach individueller Mobilität auf knapper werdenden Straßenkapazitäten gesenkt werden kann. (4) Straßennutzungsgebühren würden insbesondere Pendler dazu bringen, Alternativoptionen zur Straßennutzung zu Stoßzeiten auszuprobieren, und so die Zahl und Länge der Staus verringern.

Die Einführung von Mautsystemen mag zunächst auf große Vorbehalte der Bürger stoßen, wie Beispiele aus Bergen, Oslo und Trondheim sowie aus anderen Städten zeigen. Die zum Teil deutlichen Anstiege in der Zufriedenheit mit dem Verkehr in den drei norwegischen Städten legen jedoch nahe, dass sich diese Vorbehalte entkräften lassen, sobald die Bürger den Nutzen der Maut erkennen (Abbildung 2).

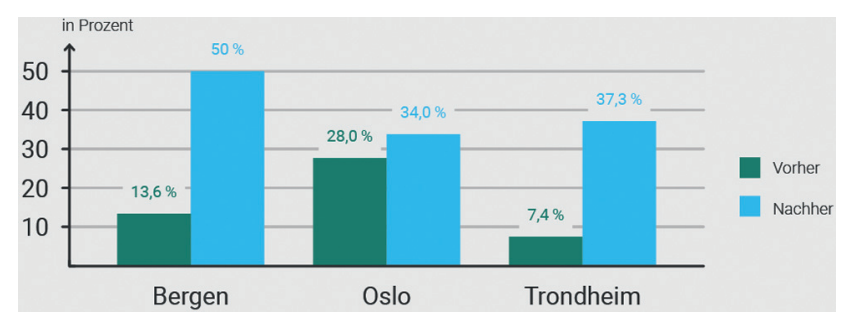

Abbildung 2: Zufriedenheit mit dem Verkehr vor und nach Einführung einer Innenstadtmaut in den norwegischen Städten Bergen, Oslo und Trondheim

Quelle: Gehlert 2009, S. 39

Analog dazu kann man in deutschen Städten wohl voraussichtlich dann eine hohe Akzeptanz einer Städte-Maut erreichen, wenn es gelingt, den Bürgern zu vermitteln, dass eine Städte-Maut nicht nur drohende Fahrverbote zu vermeiden hilft, sondern auch tatsächlich einen Ausweg aus dem Verkehrskollaps bietet. Kurzfristig wäre es sinnvoll, Modellgebiete auszuweisen, in denen ,regulative Experimente" mit Mautmodellen stattfinden können, an deren Ausgestaltung sich die Bürger umfänglich und von Anfang an beteiligen. Gleichzeitig sollten Kommunen die für Modellversuche nötige finanzielle Unterstützung erhalten. Mit einer Erprobung in Modellgebieten, beispielsweise im Rahmen der „Real-Labor-Initiative“ des Bundeswirtschaftsministeriums, begäbe man sich auf den Pfad einer modernen Verkehrspolitik und könnte zugleich helfen, sozial ungerechte und ökologisch wenig zielführende Fahrverbote zu umgehen.

Danksagung: Ich danke der Stiftung Mercator für die finanzielle Unterstützung dieses Beitrags im Rahmen des Projekts „Mobilitätsdaten für die Verkehrswende“ sowie Katja Fels, Leonard Goebel, Karen Horn und Nico Schwarzer für hilfreiche Kommentare und Anmerkungen.

\section{Literaturverzeichnis}

Achtnicht, M., M. Kesternich und B. Sturm (2018), Die „Diesel-Debatte“: Ökonomische Handlungsempfehlungen an die Politik, ZEW policy brief $3 / 2018$.

ADAC (2019), Staubilanz 2018 - Neue Rekordlängen, online verfügbar unter: https://www.adac.de/der-adac/verein/aktuelles/staubilanz/.

Beser Hugosson, M., A. Sjöberg und C. Byström (2006), Facts and Results from the Stockholm Trial, Congestion Charge Secretariat, Stockholm, online verfügbar unter: http://www.stockholmsforsoket.se/upload/Sammanfattningar/English/Final \%20Report_The \%20Stockholm \%20Trial.pdf.

Börjesson, M., J. Eliasson, M. B. Hugosson und K. Brundell-Freij (2012), The Stockholm congestion charges -5 years on. Effects, acceptability and lessons learnt, Transport Policy 20, S. 1-12. 
Börjesson, M. und I. Kristoffersson (2015), The Gothenburg congestion charge. Effects, design and politics, Transportation Research A75, S. 134-46.

Cramton, P., R. R. Geddes und A. Ockenfels (2018), Set road charges in real time to ease traffic, Nature 560, S. 23-25.

Cramton, P., R. R. Geddes und A. Ockenfels (2019), Using technology to eliminate traffic congestion, Journal of Institutional and Theoretical Economics 175(1), S. 126-39.

Downs, A. (1962), The law of peak-hour expressway congestion, Traffic Quarterly16(3), S. 393-409.

Downs, A. (1992), Stuck in Traffic: Coping with Peak-Hour Traffic Congestion, Washington, Brookings Institution Press.

Duranton, G. und M. A. Turner (2011), The fundamental law of road congestion: Evidence from US cities, American Economic Review 101(6), 2616-2652.

Eisenkopf, A. (2018), Verkehrspolitik: Stauland Deutschland? Wirtschaftsdienst 9 (2), S. 78.

FHWA, Federal Highway Administration (2019), Congestion Pricing: Examples Around the U.S. Federal Highway Administration, Washington, U.S. Department of Transportation, online verfügbar unter https://ops.fhwa.dot.gov/congestionpricing/ resources/examples_us.htm.

FHWA (2006), Congestion Pricing - A Primer. Federal Highway Administration, Washington, U.S. Department of Transportation.

Gehlert, T. (2009), Straßenbenutzungsgebühren in Städten: Akzeptanz und Mobilitätsverhalten, Verkehrspsychologie, Wiesbaden, VS Research.

Hsu, W.-T. und H. Zhang (2014), The fundamental law of highway congestion revisited: Evidence from national expressways in Japan, Journal of Urban Economics 81, S. 65-76.

INRIX (2018), INRIX 2017 Traffic Scorecard, München bleibt Deutschlands Stauhauptstadt - Hamburg und Berlin holen auf, online verfügbar unter: http://inrix.com/press-releases/scorecard2017-ger/.

Intraplan (2011), Verkehrsqualität auf deutschen Autobahnen, Studie im Auftrag des ADAC, online verfügbar unter: https://www.adac. de/_mmm/pdf/fi_verkehrsqualitaet_zusammenfassung_14_211845.pdf.

Kraftfahrtbundesamt, KBA (2019), Jahresbilanz des Fahrzeugbestandes am 1. Januar 2019, https://www.kba.de/DE/Statistik/Fahrzeuge/Bestand/bestand_node.html.

Leape, J. (2006), The London congestion charge, Journal of Economic Perspectives 20(4), S. 157-76.
Statista (2019), Teuerste Straßenbauprojekte in Deutschland bis zum Jahr 2030, online verfügbar unter: https://de.statista.com/ statistik/daten/studie/538158/umfrage/teuerste-strassenbauprojekte-in-deutschland/.

TfL, Transport for London (2019), Congestion Charge, online verfügbar unter: http://www.tfl.gov.uk/roadusers/congestioncharging/ 6723.aspx.

Peirce, S., S. Puckett, M. Petrella, P. Minnice, R. Ray und J. Lappin (2014), Effects of Full-Facility Variable Tolling on Traveler Behavior: Evidence from a Panel Study of the SR-520 Corridor in Seattle. Bericht für die Federal Highway Administration. Volpe National Transportation Systems Center, Cambridge, MA.

Vickrey, W. S. (1963), Pricing in urban and suburban transport, American Economic Review 53(2), 452-65.

Vickrey, W. S. (1969), Congestion theory and transport investment, American Economic Review 59(2), 251-60.

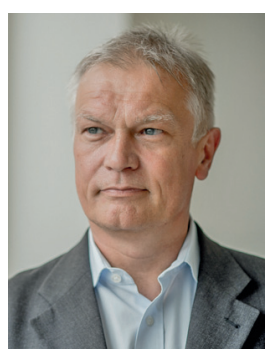

Manuel Frondel

RWI Leibniz-Institut für

Wirtschaftsforschung und Ruhr-Universität

Bochum

Hohenzollernstr. 1-3

45128 Essen

frondel@rwi-essen.de

Manuel Frondel (geb. 1964) ist seit 2003 Leiter des Kompetenzbereichs Umwelt und Ressourcen am RWI Leibniz-Institut für Wirtschaftsforschung und seit 2009 außerplanmäßiger Professor für Energieökonomik und angewandte Ökonometrie an der Ruhr-Universität Bochum. Zuvor war er am ZEW - Leibniz-Zentrum für Europäische Wirtschaftsforschung beschäftigt. Er hat Physik und Wirtschaftsingenieurwesen an der Universität Karlsruhe studiert und wurde am Lehrstuhl Ökonometrie der Universität Heidelberg promoviert. Seine Forschungsinteressen liegen im Bereich der empirischen Wirtschaftsforschung, vor allem der Anwendung statistisch-ökonometrischer Methoden auf umwelt-, ressourcen- und energie-ökonomische Fragestellungen. Manuel Frondel ist seit 2010 Fakultätsmitglied der Ruhr Graduate School in Economics (RGS Econ). 